\title{
$L R R C 17$ regulates the bone metabolism of human bone marrow mesenchymal stem cells from patients with idiopathic necrosis of femoral head through Wnt signaling pathways: A preliminary report
}

\author{
DA SONG ${ }^{1,2}$, ZHEN-SONG WU ${ }^{3}$, QI XU ${ }^{2}$, KAI WANG ${ }^{2}$, MING-TAO XU $^{2}$, \\ CHENG-ZHI HA ${ }^{2}$, CHAO ZHANG ${ }^{2}$ and DA-WEI WANG ${ }^{2}$

\footnotetext{
${ }^{1}$ Department of Orthopedics, Liaocheng People's Hospital, Cheeloo College of Medicine, Shandong University;

${ }^{2}$ Department of Orthopedics, Liaocheng People's Hospital, Liaocheng, Shandong 252000;

${ }^{3}$ Department of Joint Surgery, Zaozhuang Municipal Hospital, Zaozhuang, Shandong 277100, P.R. China
}

Received October 29, 2020; Accepted March 19, 2021

DOI: $10.3892 /$ etm.2021.10098

\begin{abstract}
Idiopathic necrosis of the femoral head (INFH) is a common disease with unknown cause. Its successful treatment relies on the repair of the necrotic bone. The application of autologous mesenchymal stem cells (MSCs) has shown great promise in saving the patients from undergoing total hip arthroplasty. Leucine-rich repeat-containing 17 (LRRC17) is less expressed in patients with femoral head necrosis and LRRC17 can inhibit bone degradation. However, it remains unknown whether $L R R C 17$ plays a role in the pathogenesis of INFH. The present study aimed to investigate the potential role and mechanism of LRRC17 in the pathogenesis and treatment of INFH. It was found that despite the similar cell morphology and MSC surface marker expressions of human bone marrow MSCs (BMSCs) isolated from patients with INFH (INFH-hBMSC) and femoral neck fracture (FNF) (FNF-hBMSC), INFH-hBMSC had higher percentage of apoptosis $(\mathrm{P}<0.05)$, as well as lower osteogenic potential and higher adipogenic potential (both $\mathrm{P}<0.05$ ). However, there was no difference in cell proliferation between FNF-hBMSC and INFH-hBMSC $(\mathrm{P}>0.05)$. It was also confirmed that
\end{abstract}

Correspondence to: Dr Da-Wei Wang, Department of Orthopedics, Liaocheng People's Hospital, 67 Dongchang West Road, Liaocheng, Shandong 252000, P.R. China

E-mail:wdw360892@163.com

Abbreviations: DMEM, Dulbecco's modified Eagle's medium; FNF, femoral neck fracture; hBMSCs, human bone marrow mesenchymal stem cells; H\&E, hematoxylin and eosin; INFH, idiopathic necrosis of the femoral head; LRRC17, leucine-rich repeat-containing 17; RT-qPCR, reverse transcription-quantitative polymerase chain reaction; shRNA, short hairpin RNA

Key words: femoral neck fracture, INFH, LRRC17, mesenchymal stem cells, Wnt signaling pathways the expression of $L R R C 17$ was lower in the bone tissue and hBMSCs from patients with INFH compared with patients with FNF $(\mathrm{P}<0.05)$. Overexpression of $L R R C 17$ promoted osteogenesis and inhibited the adipogenesis in hBMSCs, accompanied with the increase of Wnt $3 \mathrm{a}$ and $\beta$-catenin expressions, and the decrease of Wnt5a and receptor activator of nuclear factor $\kappa-\mathrm{B}$ ligand (Rankl) expressions (all, $\mathrm{P}<0.05$ ). Furthermore, knockout of LRRC17 in hBMSCs inhibited the expression levels of osteogenic and promoted adipogenic markers, while decreasing Wnt $3 \mathrm{a}$ and $\beta$-catenin expressions, and increasing Wnt5a and Rankl expressions (all, $\mathrm{P}<0.05$ ). The present preliminary study suggested that imbalanced bone metabolism may be involved in the pathogenesis of INFH. The modulation of the LRRC17 gene may delay or even restore the balance of osteogenic and adipogenic differentiation in autologous BMSCs derived from patients with INFH, providing a new target for the treatment of INFH.

\section{Introduction}

Idiopathic necrosis of the femoral head (INFH) is the osteonecrosis of the femoral head with unknown causes. It is a common disease and the number of cases is estimated at 10,000 to 20,000 cases per year in the USA $(1,2)$. This number increases every year. A recent study from Japan reported an annual incidence of 2.51 cases per 100,000 person per year (3). Early manifestations of INFH are atypical. With the progression of the disease, patients may develop collapsed femoral head, pain, shortened limbs and arthritis, eventually surgical treatments are needed. One of the available early treatment is core compression (4). It can reduce pain in patients with early stage of the disease (5). However, a significant number of patients are dissatisfied with the repair of the femoral head necrotic area (5). The other promising treatment is stem cell therapy. The necrosis of femoral head involves the local stem cell activity (6).

Human bone marrow mesenchymal stem cells (hBMSCs) are multipotent stem cells isolated from the bone marrow that 
are important for making and repairing skeletal tissues. It has strong self-renewal abilities and can undergo chondrogenic, osteogenic and adipogenic differentiation. It also maintains the balance of bone metabolism in the human skeletal system (7). Recently, the decrease in hBMSC and the change in cell behavior have been linked to the occurrence and development of osteonecrosis of the femoral head (8). This suggests that BMSC maybe a great target for INFH treatment. Wnt signaling has been implicated in controlling BMSC fate, but it has not been well investigated in hBMSC from INFH patients.

Recently, Wang et al conducted a microRNA expression profiling of BMSCs in patients with femoral head osteonecrosis compared to femoral neck fracture. They found a group of differentially expressed microRNAs and predicted their target genes. Among these, the expression of Leucine-rich repeat-containing 17 (LRRC17) was significantly lower in hBMSCs obtained from patients with femoral head osteonecrosis compared to femoral neck fracture (9). LRRC17 is a secretory protein containing five leucine-rich repeat domains. It was first characterized in bone metabolism as an inhibitor of receptor activator of nuclear factor- $\kappa \mathrm{B}$ ligand (RANKL)-induced osteoclast differentiation (10). RANKL is the ligand of the $N F-\kappa B$ receptor activator, which is an indispensable biomolecule in osteoclast activation and proliferation (10). $L R R C 17$ negatively regulates $R A N K L$ to inhibit bone degradation. Later, Kim et al demonstrated that postmenopausal women with lower $L R R C 17$ level had a 3.32-fold higher odds ratio for osteoporotic fracture and associated with a $46 \%$ higher risk of osteoporotic fracture than the group with higher LRRC17 levels, suggesting LRRC17's potential as a marker for osteoporotic fracture (11). However, the role of LRRC17 in the INFH has never been fully investigated.

In this study, we aimed to investigate the role of LRRC17 in hBMSCs derived from INFH patients. By manipulating the expression of LRRC17, we explored the potential mechanisms with a focus on the Wnt signaling pathways. This study may help us understand the effect of LRRC17 on the pathogenesis of INFH and identify a potential treatment target for INFH patients.

\section{Materials and methods}

Clinical samples. The present study was approved by the Ethics Committee of Liaocheng People's Hospital, and all patients who participated in this study provided a signed written informed consent.

Hematoxylin-eosin staining. The surgically resected femoral head was semi-dissected from the coronal face, and the appearance of the specimen and the pathology of the section were observed. A bone grain of $\sim 10 \times 10 \times 5 \mathrm{~mm}$ was taken from the necrotic area (INFH-derived) and normal area (FNF-derived) under naked eye observation. All the bone blocks were fixed in $10 \%$ formalin solution at $4^{\circ} \mathrm{C}$ for 3 days, decalcified with $10 \%$ EDTA Tris-HCl ( $\mathrm{pH}$ 7.4) buffer and changed every day at $4^{\circ} \mathrm{C}$. When acupuncturing specimen had no resistance, this indicated that the bone tissue had been decalcified completely. The specimen was embedded in paraffin and cut into sections with a thickness of 4-5 $\mu \mathrm{m}$. Sections were dewaxed using xylene for three times with 5 min each, rehydrated using gradient ethanol $(100,95,85$ and $75 \%)$, and stained with hematoxylin solution at $25^{\circ} \mathrm{C}$ for $10 \mathrm{~min}$. After that, the sections were dipped in $1 \%$ hydrochloric acid for $2-5 \mathrm{sec}, 1 \%$ ammonia for $1 \mathrm{~min}$, and then $0.5 \%$ eosin for $5-10 \mathrm{sec}$ at $25^{\circ} \mathrm{C}$. Finally, sections were dehydrated with 95 and $100 \%$ ethanol and mounted with xylene-based glue. Blocking was not performed. Sections were observed under a light microscope at a magnification of x200 and images were captured.

Bone marrow were harvested from five patients with INFH (three males and two females; average age, 61.33 \pm 7.2 years old) and five patients with femoral neck fracture (FNF; two males and three females; average age, $67.33 \pm 8.6$ years old) between February 2017 and February 2018, who received femoral head replacement in the hospital. According to the Ficat staging system (12), all patients with INFH were at stages III or IV. The exclusion criteria were: i) Medical history of cancer; ii) other metabolic bone disease; iii) previous hip surgery or infectious disease; and iv) long-term use of hormones and alcohol. hBMSCs harvested from five patients with INFH or FNF (INFH-hBMSC or FNF-hBMSC) were pooled together for following experiments.

All experimental procedures were conducted in strict conformity with the work described, and carried out in accordance with the Code of Ethics of the World Medical Association (Declaration of Helsinki) for experiments involving humans.

Isolation and culture of hBMSCs. The hBMSCs were isolated by subjecting to Ficoll $(1.077 \mathrm{~g} / \mathrm{ml}$; Tianjin Haoyang Biological Products Technology Co., Ltd.) density gradient separation. The mononuclear fraction was collected and plated in T-25 flasks (Corning, Inc.) using complete DMEM/F-12 containing $10 \%$ fetal bovine serum and $1 \%$ penicillin and streptomycin (Gibco; Thermo Fisher Scientific, Inc.) medium at $37^{\circ} \mathrm{C}$ with $5 \% \mathrm{CO}_{2}$. After three days, the medium was changed to remove non-adherent cells. Then, the media was changed every three days. When the primary cells reached 70-80\% confluence, the cells were passaged by trypsinization (Hyclone; Cyvita), and cultured based on the aforementioned method.

Flow cytometry. The cell-specific surface markers were examined by flow cytometry. The hBMSCs were dissociated and fixed in $70 \%$ ethanol at $4^{\circ} \mathrm{C}$ for $24 \mathrm{~h}$. Anti-CD34-FITC (cat. no. 560942), anti-CD44-FITC (cat. no. 560977), anti-CD45-FITC (cat. no. 560976) and anti-CD90-FITC (cat. no. 561969) (all ready to use; BD Biosciences PharMingen) antibodies were used and incubated at $25^{\circ} \mathrm{C}$ for $20 \mathrm{~min}$. The data analysis was conducted using the BD FACSDiva software (version. 8.0.1; BD Biosciences).

Osteogenic induction and evaluation. To induce osteogenic differentiation, hBMSCs were incubated in DMEM supplemented with dexamethasone (100 nM), $\beta$-glycerophosphate $(10 \mathrm{mM})$ and ascorbic acid 2-phosphate $(200 \mu \mathrm{M})$ (all from Sigma-Aldrich; Merck KGaA), and $10 \%$ fetal bovine serum (Hyclone; Cyvita). The medium was changed every three days. After osteogenic induction for 14 days, cells were stained with $2 \%$ alizarin red S (Sigma-Aldrich; Merck KGaA) at $25^{\circ} \mathrm{C}$ for $15 \mathrm{~min}$ to evaluate the calcium deposition. Matrix mineral-bound staining was detected as a bright orange-red color under a light microscope (magnification, x200). 
Adipogenic induction and evaluation. To induce adipogenic differentiation, lipid induction A solution was prepared by supplementing DMEM with dexamethasone $(1 \mu \mathrm{M})$, 3 -isobutyl-1-methylxanthine $(0.5 \mathrm{mM})$, insulin $(10 \mu \mathrm{g} / \mathrm{ml})$, rosiglitazone $(0.5 \mu \mathrm{M})$ and indomethacin $(100 \mu \mathrm{M})$ (all from Sigma-Aldrich; Merck KGaA) and $10 \%$ fetal bovine serum (Hyclone; Cyvita). Then, B solution was prepared by adding insulin $(20 \mu \mathrm{g} / \mathrm{ml})$ to the DMEM and $10 \%$ fetal bovine serum. Subsequently, $2 \times 10^{5} / \mathrm{ml}$ hBMSCs were incubated with the A solution for three days, and this was switched to the B solution for one day. This was repeated for four times. In order to evaluate the adipogenesis, oil red O (Sigma-Aldrich; Merck KGaA) staining was performed at $25^{\circ} \mathrm{C}$ for $15 \mathrm{~min}$ and observed under a light microscope at a magnification of x 200 .

Cell transfection. The INFH-hBMSCs were randomly divided into four groups: LRRC17 overexpression group and its vector control group, and LRRC17 knockout group and its negative control group. For the overexpression or knockout, INFH-hBMSCs were transfected with lentiviral particles of GV492-LRRC17 or GV493-LRRC17 short hairpin (sh)RNA, and the respective control vectors. The quantity of lentiviral plasmid used for transfection was $9 \mu \mathrm{g}$. The ratio of the lentiviral plasmid: Packaging vector: Envelope was 9:13:4. The duration of transfection into cells was 3 days and the multiplicity of infection (MOI) was $70 \%$.

The lentiviral supernatants that contained lentiviral vectors for $L R R C 17$ overexpression or knockout, and the respective controls were purchased from Shanghai GeneChem Co., Ltd.

Positive clones were selected with $200 \mathrm{ng} / \mathrm{ml}$ of puromycin for three days. After five days, the infection efficiency was observed under a fluorescence microscope (cat. no. CKX71; Olympus Corporation). When the transduction was over, the subsequent experiments were performed immediately.

Dickkopf-related protein 1 (DKK1) and Wnt inhibition treatment. INFH-hBMSCs were seeded at $1 \times 10^{3}$ in a 6 -well plate, and randomly divided into four groups: LRRC17 overexpression treatment with or without $100 \mathrm{ng} / \mathrm{ml}$ DKK1 (MedChemExpress) treatment, and LRRC17-knockout cells with or without $10 \mu \mathrm{M}$ SP600125 (Beyotime Institute of Biotechnology). Then, cells were transfected for the LRRC17 overexpression or knockout cells at $24 \mathrm{~h}$ after plating, followed by DKK1 or SP600125 treatment after 48 h. Cells were harvested after $24 \mathrm{~h}$ for downstream analysis.

In vitro cell proliferation assay. In order to generate the standard curve, hBMSCs were trypsinized and seeded at $2 \times 10^{3}$, $4 \times 10^{3}, 8 \times 10^{3}, 1.0 \times 10^{4}, 1.2 \times 10^{4}, 1.6 \times 10^{4}, 2.0 \times 10^{4}$ and $2.4 \times 10^{4}$ cells per well in a 96 -well plate. Each well was repeated for three times. After 2 h, cells were incubated with Cell Counting Kit-8 (CCK-8) (Dojindo Molecular Technologies, Inc.) for another $2 \mathrm{~h}$ at $25^{\circ} \mathrm{C}$ and detected using an enzyme-labeling instrument.

The INFH-hBMSCs or FNF-hBMSCs were trypsinized, and $5 \times 10^{3}$ cells per well were plated in a 96 -well plate. For each of the following six days, cells were incubated with CCK-8 for $2 \mathrm{~h}$ at $37^{\circ} \mathrm{C}$ and detected using an enzyme labeling instrument.

Cell apoptosis assay. Annexin V-FITC (BD Biosciences) was used to evaluate the apoptosis in INFH-hBMSCs or
FNF-hBMSCs. Then, $1 \times 10^{6}$ cells from each group were resuspended with $1 \mathrm{X}$ binding buffer, and $5 \mu \mathrm{l}$ of Annexin V-FITC was added and incubated for $15 \mathrm{~min}$ at room temperature in the dark. Propidium iodide (PI) staining with a volume of $5 \mu \mathrm{l}$ was added at $5 \mathrm{~min}$ before detection. Then, cells were analyzed by flow cytometry (Becton, Dickinson and Company).

Reverse transcription-quantitative polymerase chain reaction (RT-qPCR). TRIzol RNA isolation reagents (Thermo Fisher Scientific, Inc.) was used to extract the total RNA. A NanoDrop 2000 Spectrophotometer (Thermo Fisher Scientific, Inc.) was used to measure the concentration and purity of the total RNA. Then, $1 \mu \mathrm{g}$ RNA was converted to cDNA using a PrimeScript $^{\mathrm{TM}}$ RT reagent kit (Takara Bio, Inc.) with a gDNA Eraser at $37^{\circ} \mathrm{C}$ for $15 \mathrm{~min}$, then $85^{\circ} \mathrm{C}$ for $5 \mathrm{sec}$ and $4^{\circ} \mathrm{C}$ continuously, and amplified in the ABI 7500 Sequence Detection System (Applied Biosystems; Thermo Fisher Scientific, Inc.) using a SYBR ${ }^{\circledR}$ Premix Ex Taq ${ }^{\mathrm{TM}}$ II kit (all obtained from Takara Biotechnology Co., Ltd.). The thermal profile of the RT-qPCR was: Pre-incubation at $95^{\circ} \mathrm{C}$ for $30 \mathrm{sec}$ for one cycle, followed by 40 cycles incubated at $95^{\circ} \mathrm{C}$ for $5 \mathrm{sec}$, and $60^{\circ} \mathrm{C}$ for $34 \mathrm{sec}$. The polymerase chain reaction (PCR) primers were synthesized by Sangon Biotech Co., Ltd. The primer sequences are summarized in Table I. The housekeeping gene GAPDH was used as an internal control. The relative expression levels of each gene were calculated using $2^{-\Delta \Delta \mathrm{Cq}}(13)$. Each experiment was evaluated by the same PCR reactions for three times.

Western blotting. The protein expression was measured after transfecting hBMSCs with lentivirus that contained the LRRC17 gene. The expression levels of osteoprotegerin (OPG), bone morphogenetic protein 2 (BMP2), peroxisome proliferator-activated receptor $\gamma(\operatorname{PPAR} \gamma)$, CCAAT/enhancer-binding protein $\alpha(\mathrm{CEBP} \alpha)$, Wnt3a, $\beta$-catenin, Wnt5a and Rank1 proteins were measured for all groups of hBMSCs.

PMSF was used to lyse the cell precipitation. The cell lysates were centrifuged at $10,000 \mathrm{x}$ for $4 \mathrm{~min}$ at $4^{\circ} \mathrm{C}$, and the $\mathrm{BCA}$ protein assay kit was used to measure the protein concentrations (all were obtained from Beyotime Institute of Biotechnology). The same amount of protein $(10 \mu \mathrm{g})$ and Prestained Protein Ladder $(4 \mu \mathrm{l})$ were injected into the $10 \%$ sodium dodecyl sulfate-polyacrylamide gel electrophoresis lanes, and electrophoresis was performed for $1 \mathrm{~h}$ at $100 \mathrm{~V}$. Then, the proteins were transferred onto polyvinylidene fluoride membranes (EMD Millipore) for $1.5 \mathrm{~h}$ at $200 \mathrm{~mA}$. Next, 5\% skimmed milk (dissolved in TBST) was used to block the non-specific binding membranes at room temperature for $1 \mathrm{~h}$. Then, the membranes were incubated with the appropriate primary antibodies overnight at $4^{\circ} \mathrm{C}$ : Anti-LRRC17 (1:600), anti-CEBP $\alpha$ (1:750), anti- $\beta$-catenin $(1: 30,000)$ (all obtained from Proteintech Group, Inc.); anti-OPG (1:1,000; ABclonal Biotech Co., Ltd.); anti-Wnt5a (1:1,000), anti-BMP2 (1:1,000), anti-PPAR $\gamma$ (1:1,000), anti-RANKL (1:700), anti-Wnt3a (1:1,000), mouse monoclonal anti- $\beta$-actin antibody $(1: 10,000)$ (all obtained from Beyotime Institute of Biotechnology). TBST was used to wash the membranes three times, for $10 \mathrm{~min}$ each. The membranes were incubated with species-specific horseradish peroxidase coupled with the secondary antibodies (goat anti-mouse or anti-rabbit IgG; cat. nos. SA00001-1 and SA00001-2, respectively) at 1:5,000 for $1 \mathrm{~h}$ at room temperature. After washing 
Table I. Sequences of primers.

\begin{tabular}{|c|c|c|c|}
\hline Gene & Refseq accession number & Direction & Sequence, $5^{\prime}$ to $3^{\prime}$ \\
\hline LRRC17 & NM_001031692 & $\begin{array}{l}\text { Forward } \\
\text { Reverse }\end{array}$ & $\begin{array}{l}\text { AAAGTGCCAAACAACATCCCT } \\
\text { TGGGTCGAAGTTGGTTGATTTT }\end{array}$ \\
\hline OPG & NM_002546.4 & $\begin{array}{l}\text { Forward } \\
\text { Reverse }\end{array}$ & $\begin{array}{l}\text { GCCCCTTGCCCTGACCACTAC } \\
\text { TGCGATTGCACTCCTGCTTGAC }\end{array}$ \\
\hline BMP2 & NM_001200.4 & $\begin{array}{l}\text { Forward } \\
\text { Reverse }\end{array}$ & $\begin{array}{l}\text { GTCCTGAGCGAGTTCGAGTTGC } \\
\text { GTGGTCTGGGGCGGGTGAG }\end{array}$ \\
\hline $\operatorname{CEBP} \alpha$ & NM_004364.4 & $\begin{array}{l}\text { Forward } \\
\text { Reverse }\end{array}$ & $\begin{array}{l}\text { TCGGTGGACAAGAACAGCAACG } \\
\text { GGCGGTCATTGTCACTGGTCAG }\end{array}$ \\
\hline $\operatorname{PPAR} \gamma$ & NM_015869.4 & $\begin{array}{l}\text { Forward } \\
\text { Reverse }\end{array}$ & $\begin{array}{l}\text { GCTGAATCCAGAGTCCGCTGAC } \\
\text { ATCGCCCTCGCCTTTGCTTTG }\end{array}$ \\
\hline Wnt3a & NM_033131.4 & $\begin{array}{l}\text { Forward } \\
\text { Reverse }\end{array}$ & $\begin{array}{l}\text { TCGGAGATGGTGGTGGAGAAGC } \\
\text { GGGTTGGGCTCGCAGAAGTTG }\end{array}$ \\
\hline Wnt5a & NM_003392.5 & $\begin{array}{l}\text { Forward } \\
\text { Reverse }\end{array}$ & $\begin{array}{l}\text { GACTTCCGCAAGGTGGGTGATG } \\
\text { GTCTTGTGTGGTGGGCGAGTTG }\end{array}$ \\
\hline$\beta$-catenin & NM_001904.4 & $\begin{array}{l}\text { Forward } \\
\text { Reverse }\end{array}$ & $\begin{array}{l}\text { TACTGTCCTTCGGGCTGGTGAC } \\
\text { GCTTCTTGGTGTCGGCTGGTC }\end{array}$ \\
\hline Rankl & NM_003701.4 & $\begin{array}{l}\text { Forward } \\
\text { Reverse }\end{array}$ & $\begin{array}{l}\text { CAGCATCGCTCTGTTCCTGTA } \\
\text { CTGCGTTTTCATGGAGTCTCA }\end{array}$ \\
\hline GAPDH & NM_002046.7 & $\begin{array}{l}\text { Forward } \\
\text { Reverse }\end{array}$ & $\begin{array}{l}\text { CGGAGTCAACGGATTTGGTCGTAT } \\
\text { AGCCTTCTCCATGGTGGTGAAGAC }\end{array}$ \\
\hline
\end{tabular}

for $30 \mathrm{~min}$, an ECL western blotting kit (Beyotime Institute of Biotechnology) was used to develop the blots. The quantification of the protein bands was visualized using the AlphaView analysis system (ProteinSimple). The $\beta$-actin antibody was used as the protein loading control. The LRRC17, CEBP $\alpha$, $\beta$-catenin, OPG, Wnt5a, PPAR $\gamma$, BMP2, RANKL and Wnt3a protein expression was normalized against $\beta$-actin.

Statistical analysis. The data are presented as mean \pm standard deviation (SD), and at least three independent experiments were performed. The quantitative data was collected and statistically analyzed using the SPSS 20.0 software (IBM Corp.) by one-way ANOVA between two groups followed by SNK-q and LSD test. The level of statistical significance was set at $\mathrm{P}<0.05$.

\section{Results}

Isolation and culture of hBMSCs from patients with FNF or $I N F H$. Femoral head tissues were harvested from five patients with FNF and five patients with INFH (representatively shown in Fig. 1A). The hematoxylin and eosin (H\&E) staining of femoral head tissues suggested that the destruction of the bone structure in INFH patients was more severe compared with that of patients with FNF.

The hBMSCs were isolated from the bone marrow of patients with FNF and INFH and pooled together respectively for downstream analysis. INFH-hBMSCs or FNF-hBMSCs were characterized with surface markers. The cell morphology revealed that the hBMSCs from both groups were as follows:
Spindle-shaped, had a long fusiform, uniform in size and easily adherent to the plate, arranged in a certain direction, and rich in cytoplasm with a strong refraction (Fig. 1B).

Characterization of hBMSCs by surface marker expression and multi-lineage differentiation. The expression levels of MSC surface markers (CD44 and CD90) and hematopoietic markers (CD45 and CD34) were analyzed in hBMSCs from both groups by flow cytometry (14). The results showed that hBMSCs from both INFH and FNF groups consistently expressed CD44 and CD90 and were negative for CD45 and CD34 (Fig. 1C).

In order to evaluate the adipogenic differentiation, the lipid droplet accumulation was assessed by oil red $\mathrm{O}$ staining. The present quantification data revealed that the FNF-hBMSC-induced lipid droplets were smaller and fewer in number compared with those from INFH-hBMSCs $(\mathrm{P}<0.05$; Fig. 1D).

After incubation in the osteogenic induction medium for 14 days, calcium nodules were observed through alizarin red staining, and these were further quantified. The present data show that calcium deposition was significantly higher in the FNF-hBMSCs compared with the INFH-hBMSCs $(\mathrm{P}<0.05$; Fig. 1D). These data suggested that INFH-hBMSCs had a diminished ability for osteogenic differentiation and increased potential for adipogenic differentiation.

Cell proliferation and apoptosis analysis of hBMSCs from patients with INFH or FNF. Cell proliferation was further analyzed using the classic CCK- 8 assay. Both groups of cells 

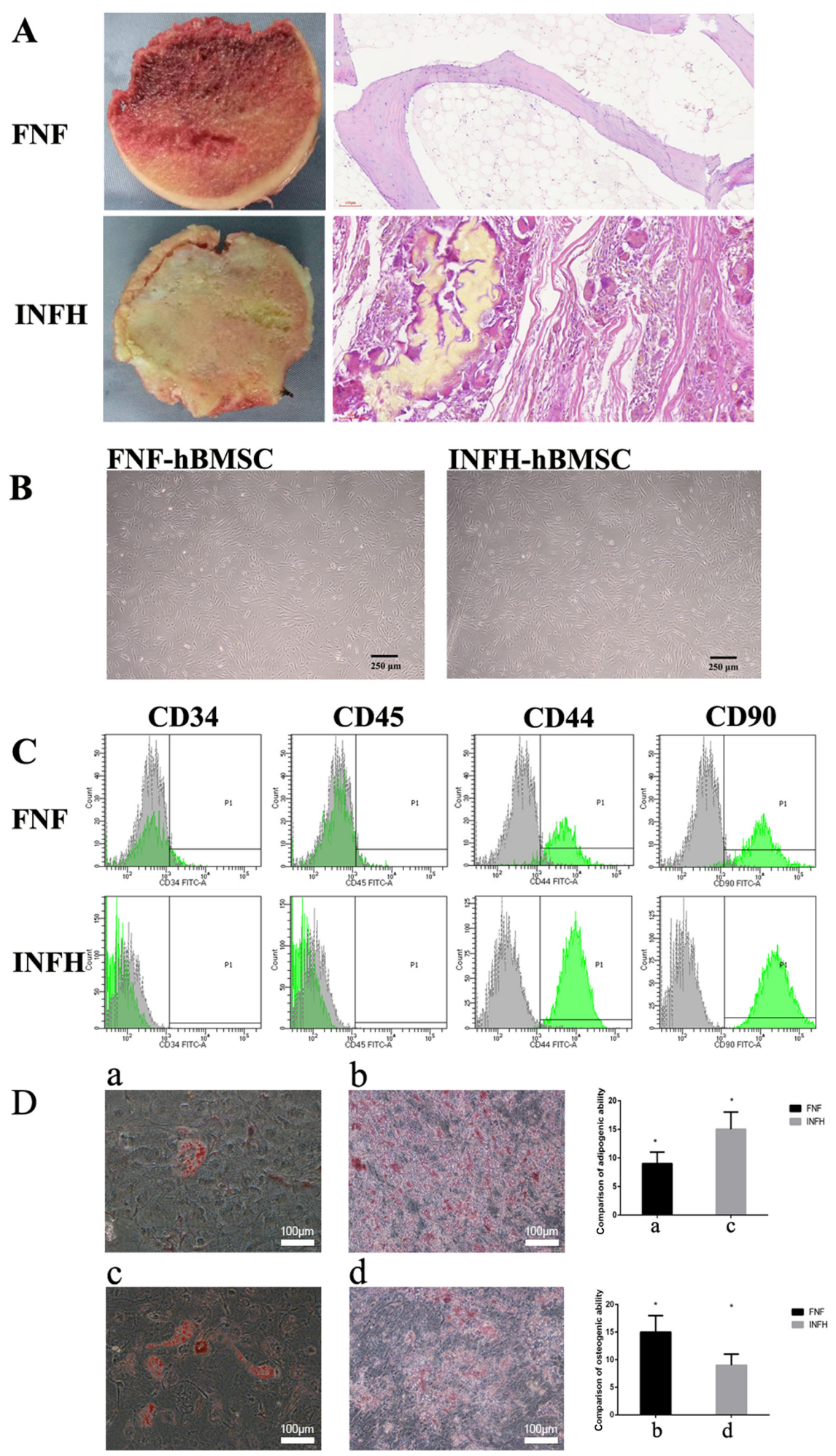

Figure 1. Characterization of hBMSCs from patients with INFH or FNF. (A) The longitudinal section and hematoxylin and eosin staining of femoral head tissues in patients with FNF or INFH. (B) The primary hBMSCs for INFH and FNF, respectively. (C) The characterization of hBMSC surface markers in patients with FNF or INFH. (D) The two images on the left represent the comparison of hBMSC adipogenic induction, and the differentiation between patients with FNF (a) and patients with INFH (c). The two images on the right represent the comparison of the osteogenic differentiation of hBMSCs in patients with FNF (b) and patients with INFH (d). Scale bar, $100 \mu \mathrm{m}$. hBMSCs, human bone marrow mesenchymal stem cells; INFH, idiopathic necrosis of the femoral head; FNF, femoral neck fracture.

were seeded at the same density on day 0 and cultured for 6 days. After generating a standard curve with $\mathrm{R}^{2}=0.9964$, the CCK-8 levels of each sample was measured. The data showed that hBMSCs from both groups continued to proliferate during the culture. The cell number of FNF-hBMSCs was comparable to INFH-hBMSCs for the first 3 days, then became higher from day 4 with a statistically significant difference observed on day 4 ( $\mathrm{P}<0.05 ;$ Fig. $2 \mathrm{~A})$. 
A

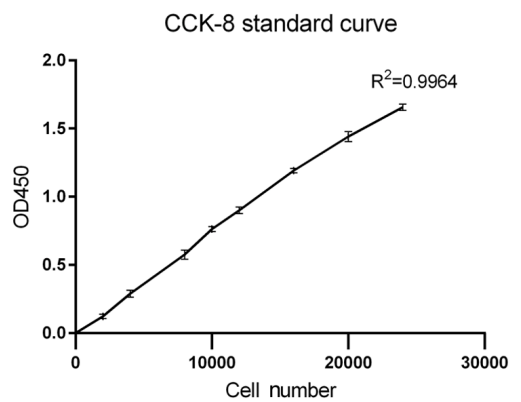

Cell growth

CCK-8 proliferation assay

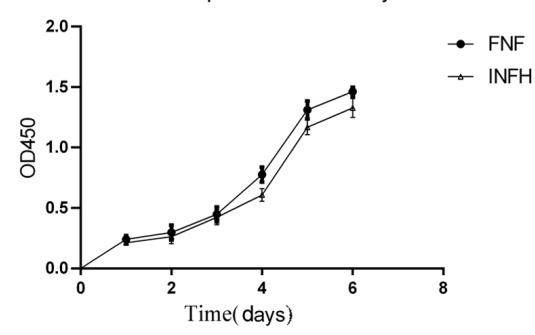

\section{B}
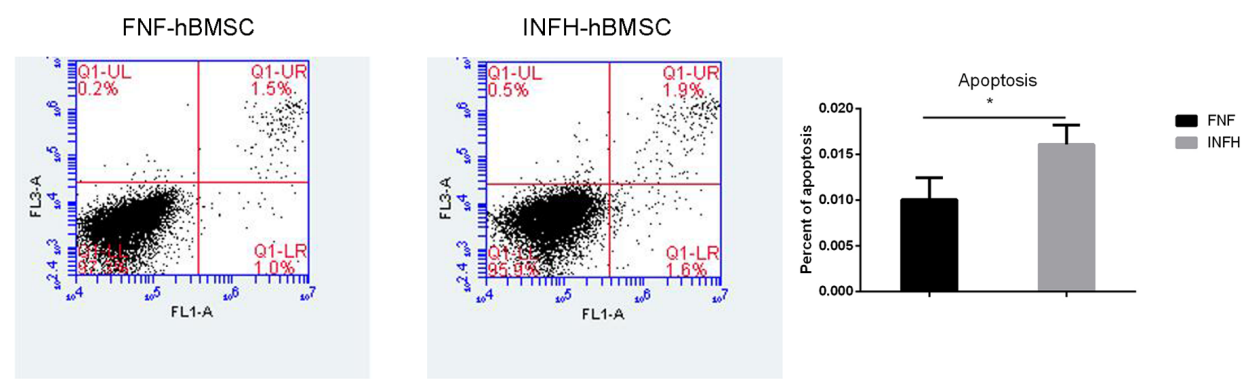

C

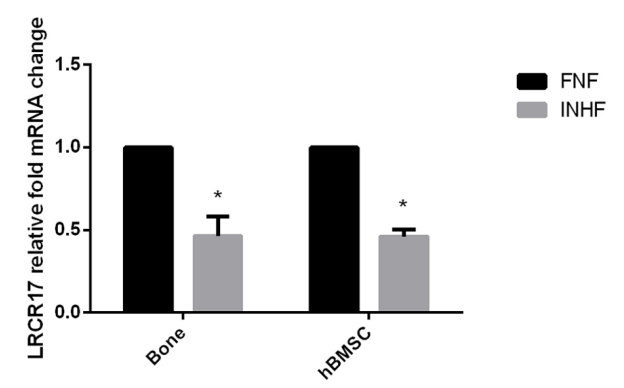

Figure 2. Cell proliferation and apoptosis in hBMSCs from patients with INFH or FNF. (A) The left presents the standard proliferation curve of patients with FNF; the right presents the comparison of hBMSC proliferation curves between patients with FNF and INFH. (B) The comparison of hBMSC apoptosis in patients with FNF (1\%) or INFH (1.6\%) (FNF, 0.01 \pm 0.002386 ; INFH, 0.016 $\pm 0.002111 ; \mathrm{P}=0.0310$ ). (C) Comparison of the mRNA expression of $L R R C 17$ in femoral head tissues and hBMSCs of patients with FNF or INFH. "P<0.05 vs. FNF. hBMSCs, human bone marrow mesenchymal stem cells; INFH, idiopathic necrosis of the femoral head; FNF, femoral neck fracture.

hBMSCs from both INFH and FNF groups did not have high level of cell apoptosis, despite that the number of cells undergoing apoptosis in INFH-hBMSCs was higher compared with those from FNF-hBMSCs (FNF, 1.0\%; INFH, 1.6\%; $\mathrm{P}<0.05$; Fig. 2B). These data suggest that INFH-hBMSCs have decreased proliferative ability and tendency to undergo apoptosis.

LRRC17 expression in the bone and hBMSCs of patients with INFH or FNF. Next, the expression of $L R R C 17$ was examined in the bone tissues and hBMSCs in each patient. It was found that the expression of $L R R C 17$ was 2.17 -fold higher on average in the bone tissue of patients with FNF compared with that from patients with INFH. Similarly, the mRNA expression of LRRC17 in the FNF-hBMSCs was 2.38-fold higher compared with that of the INFH-hBMSCs (Fig. 2C). The aforementioned data confirmed the finding presented by Wang et al (9).

LRRC17 overexpression inhibits adipogenic differentiation and promotes the osteogenic differentiation of INFH-hBMSCs. To investigate the role of LRRC17 in the pathogenesis of INFH, LRRC17 was first overexpressed in the INFH-hBMSCs, and the adipogenic and osteogenic differentiation abilities were assessed. The results showed that the mRNA levels of LRRC17 increased 5.4-fold in the $L R R C 17$-overexpressed group, as well as the osteogenic differentiation marker genes $O P G$ and $B M P 2$, which increased by 6.1- and 6.6-fold respectively, compared with its vector control group. The aforementioned changes were also confirmed at the protein level using western blotting. The protein expression of LRRC17, BMP2 and OPG was increased by 1.8-, 1.6- and 2.7-fold, respectively. Meanwhile, the mRNA expression of adipogenic markers $P P A R G$ and $C E B P \alpha$ decreased by 6.7- and 10.0-fold, respectively, in the $L R R C 17$ overexpression group compared with the control group; similarly, their protein expression levels were decreased by 1.5 - and 1.8-fold, respectively (Fig. 3A).

The knockout of LRRC17 gene expression in hBMSCs was performed using shRNA. The data showed that the $L R R C 17$ mRNA expression was decreased by 5.5 -fold with LRRC17 knockout. $O P G$ and $B M P 2$ expression levels decreased by 10.0 - and 10.0 -fold, respectively, in the knockout group, compared with the control; consistently, the protein expression levels were also decreased by 1.7-, 
A
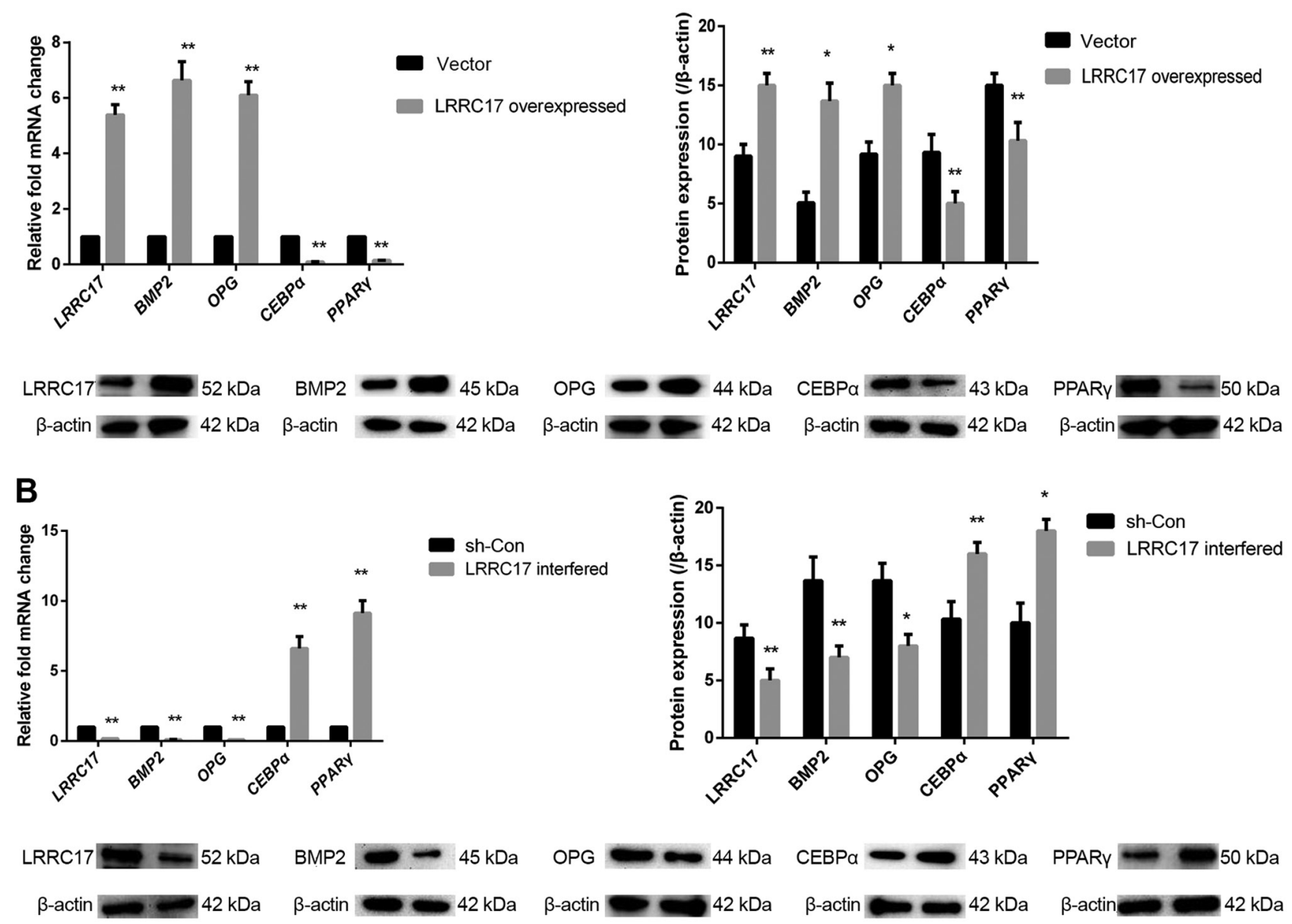

Figure 3. Effect of LRRC17 expression on osteogenic and adipogenic differentiation of INFH-hBMSCs. (A and B) Changes in LRRC17, BMP2, OPG, CEBP $\alpha$, PPAR $\gamma$ mRNA and protein expression after the overexpression and interference with LRRC17. ${ }^{*} \mathrm{P}<0.05,{ }^{* *} \mathrm{P}<0.01 \mathrm{vs}$. respective control. hBMSCs, human bone marrow mesenchymal stem cells; INFH, idiopathic necrosis of the femoral head; LRRC17, leucine-rich repeat-containing 17; OPG, osteoprotegerin; BMP2, bone morphogenetic protein 2; PPAR $\gamma$, peroxisome proliferator-activated receptor $\gamma$; CEBP $\alpha$, CCAAT/enhancer-binding protein $\alpha$; sh-, short hairpin RNA; con-, control.

1.7- and 1.9-fold, respectively. Moreover, the mRNA expression of $P P A R G$ and $C E B P \alpha$ was increased by $9.1-$ and 6.6-fold, respectively, in the knockout group compared with its negative control group, and the protein expression was increased by 1.8 - and 1.6-fold, respectively (Fig. 3B). This suggests that LRRC17 promotes osteogenesis and inhibits adipogenesis in INFH-hBMSCs.

LRRC17 regulates BMSC through Wnt signaling pathways. Since both canonical and non-canonical Wnt pathways have been shown to regulate osteogenesis and adipogenesis in BMSCs, the involvement of these pathways were further investigated to dissect the mechanisms of how LRRC17 regulates the multilineage differentiation in INFH-hBMSCs. Upon the overexpression of LRRC17, the mRNA levels of WNT3A and CTNNB1 increased by 3.3- and 8.8-fold, respectively; their protein expression levels were also increased by 2.5- and 2.3-fold. On the contrary, the mRNA levels of WNT5A and RANKL decreased by 4.0 - and 2.9-fold, respectively, due to $L R R C 17$ overexpression, and their protein expression decreased by 2.0 - and 1.9-fold, respectively (Fig. 4A).
Meanwhile, the mRNA expression of WNT3A and CTNNB1 was decreased by 2.9- and 9.9-fold, respectively, with $L R R C 17$ knockout; their protein expression levels decreased by 1.9- and 1.6-fold, respectively. The mRNA expression levels of WNT5A and RANKL were increased by 4.5- and 4.0-fold, respectively, due to $L R R C 17$ knockout. Correspondingly, the protein expression levels were increased by 2.0- and 1.7-fold, respectively (Fig. 4B). This suggests that both Wnt3a- and Wnt5a-mediated signaling pathways are involved in the regulation of hBMSC differentiation by LRRC17.

To further confirm the role of Wnt signaling pathways in INFH-hBMSCs, the canonical Wnt pathway inhibitor Dkk1 was incubated with LRRC17-overexpressed cells and the protein levels of Wnt3a and $\beta$-catenin were evaluated. The results confirmed that the expression of Wnt3a and $\beta$-catenin was decreased by 1.8- and 1.7-fold, respectively, with the addition of Dkk1 (Fig.4C). On the other hand, the protein expression levels of Wnt5a and Rankl in the LRRC17-knockout group with SP600125 treatment was decreased by 1.7- and 1.5-fold, respectively. These data further confirm the involvement of Wnt signaling in LRRC17 in regulating bone metabolism, and 
A
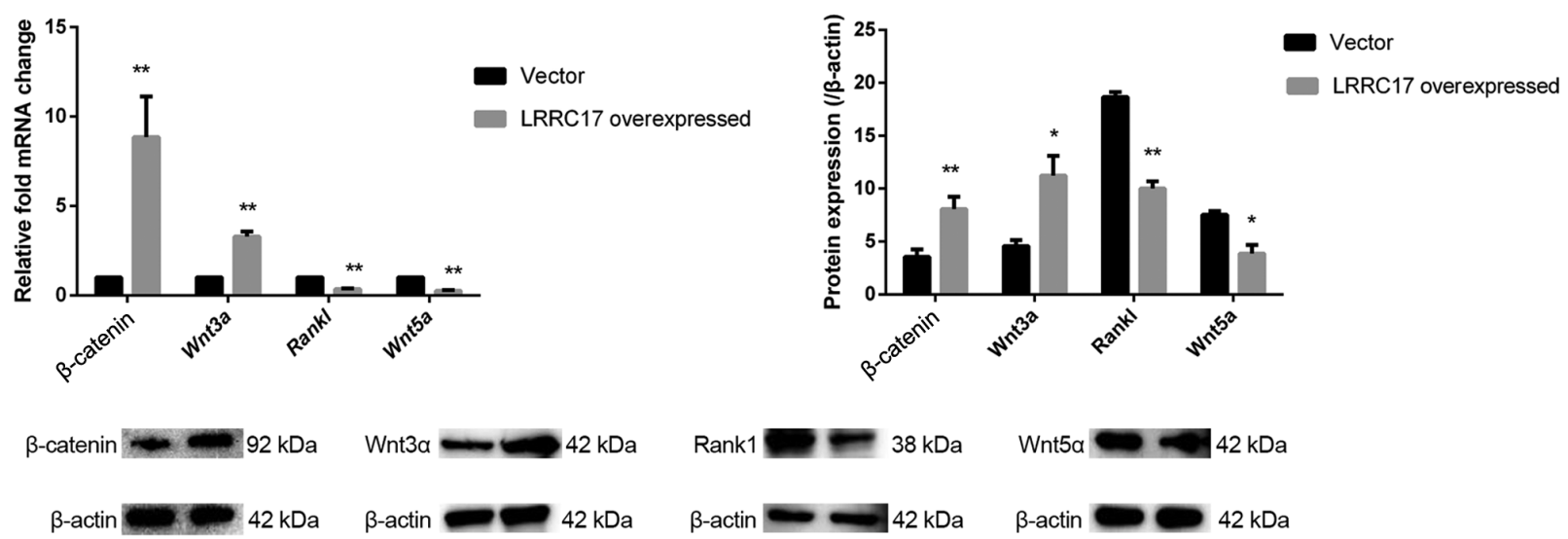

B
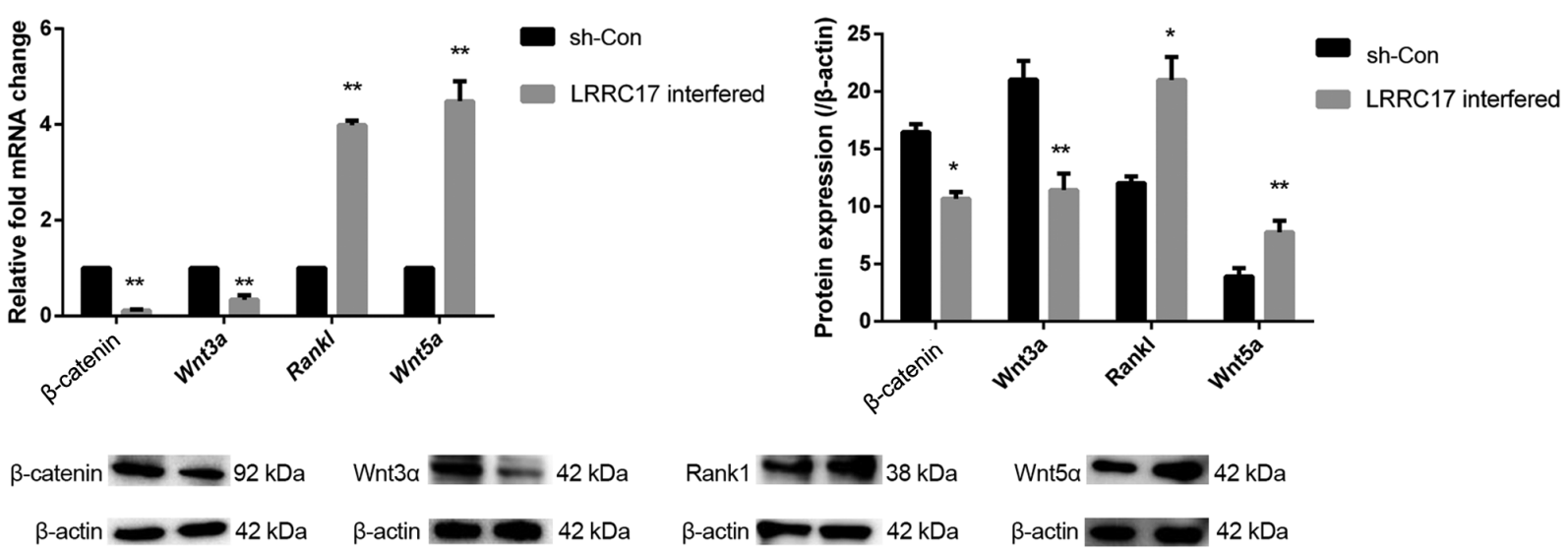

C

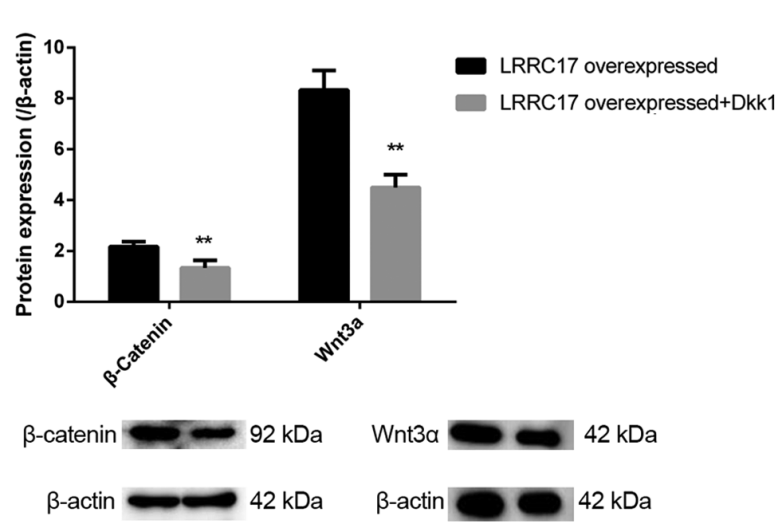

D

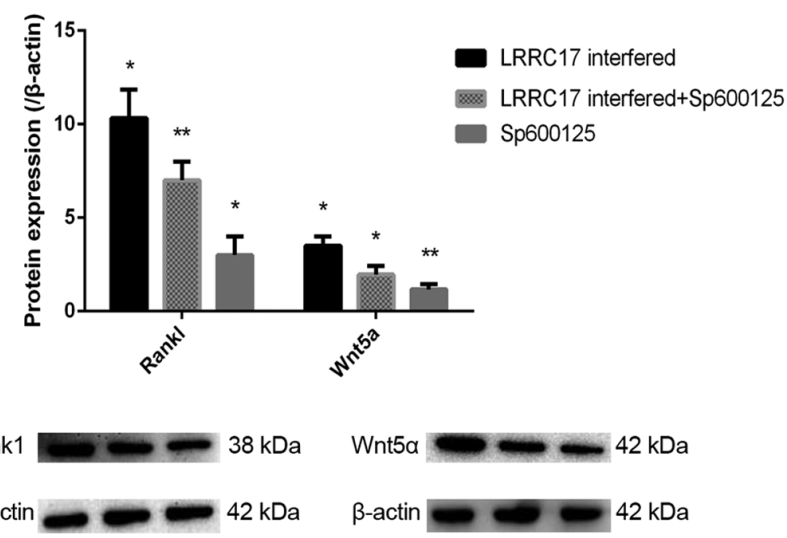

Figure 4. Effect of LRRC17 on Wnt signaling in the INFH-hBMSCs. (A and B) Changes in $\beta$-catenin, Wnt3a, Rankl and Wnt5a mRNA and protein expression after the overexpression and interference with LRRC17. (C) Changes in $\beta$-catenin and Wnt3a protein expression after the overexpression of $L R R C 17$ and the addition of DKK1. (D) Changes in Rank1 and Wnt5a protein expression after the interference of $L R R C 17$ and the addition of Sp600125. ${ }^{*}<0.05$, ${ }^{* *} \mathrm{P}<0.01$ vs. the control groups. hBMSCs, human bone marrow mesenchymal stem cells; INFH, idiopathic necrosis of the femoral head; LRRC17, leucine-rich repeat-containing 17; Rank1, receptor activator of nuclear factor $\kappa-B$ ligand; DKK1, Dickkopf-related protein 1; sh-, short hairpin RNA; con-, control.

provides the target of promoting osteogenesis in hBMSCs from patients with INFH (Fig. 4D).

\section{Discussion}

The present study characterized the morphology, surface marker expression, cell proliferation and multi-lineage differentiation abilities of hBMSCs from patient with INFH, and demonstrated that $L R R C 17$ may be a pivotal regulator of bone metabolism in the pathogenesis of INFH. The present study data suggested that INFH-hBMSCs were similar in cell morphology, surface marker expressions, but with enhanced adipogenic differentiation and decreased osteogenic differentiation abilities compared with FNF-hBMSCs. INFH-hBMSCs 
also expressed lower levels of $L R R C 17$ and were highly apoptotic compared with FNF-hBMSCs. The overexpression of LRRC17 promoted osteogenesis, and inhibited adipogenesis, while the knockout of $L R R C 17$ reversed these changes in the INFH-hBMSCs; this may be mediated through both canonical and non-canonical Wnt signaling pathways. The results suggested that $L R R C 17$ may be a potential target for the stem cell therapeutic treatment of INFH.

The present study data demonstrated that $L R R C 17$ has an important role in the pathogenesis of INFH. Abnormal bone metabolism is part of the pathogenesis of femoral head osteonecrosis (15). During INFH, the proliferative and osteogenic differentiation abilities of hBMSCs decrease. This diminishes the bone-repair ability of the body, further leading to subchondral bone weakening, stress fracture, and even articular surface collapse. Consistently, it was also found that the osteogenic differentiation of INFH-hBMSCs was significantly decreased and adipogenic differentiation ability was increased compared with FNF-hBMSCs. LRRC17 expression was significantly lower in patients with INFH. Overexpression of $L R R C 17$ promoted osteogenesis, and inhibited the adipogenesis of INFH-hBMSCs. Therefore, targeting $L R R C 17$ provides a promising therapeutic target for stem cell treatment of patients with INFH.

Previous studies have confirmed that Wnt signaling pathways participate in regulating MSC self-renewal and osteogenic differentiation, and maintaining the balance between osteoblast and osteoclast differentiation $(16,17)$. This has important clinical significance for bone injury repair, tissue renewal, and intra-articular homeostasis (18). Wnt signal pathways are evolutionarily conserved, and can be mainly classified as follows: Canonical- or Wnt/ $\beta$-catenin-dependent pathway, and the non-canonical or $\beta$-catenin-independent pathway. These two pathways intersect with each other, and their association is very complicated, and has not been fully elaborated currently (19). The members of the Wnt family are involved in bone regeneration, including the proliferation and differentiation of osteoblasts, and their progenitors (20). The Wnt/ $\beta$-catenin signaling stimulates the generation of osteoblasts by promoting the osteogenic differentiation of MSCs, while suppressing the other two lineages differentiation (21). It has been shown that MSC osteogenesis can be induced by increasing the transcription of $\beta$-catenin (22). The overexpression of the $\mathrm{Wnt} / \beta$-catenin signal in periosteal cells can increase intramembranous ossification and endochondral ossification $(23,24)$.

Wnt3a can regulate the proliferation of differentiated osteoblasts and their progenitors, and promote the differentiation of MSCs into osteoblasts under appropriate conditions (25). In addition, Wnt3a binds to the Frizzled and LRP5 or LRP6 receptor complex, inhibits GSK-3 $\beta$, and promotes the accumulation of $\beta$-catenin in osteoblasts $(26,27)$. The accumulated $\beta$-catenin translocates into the nucleus, and together with TCF/LEF, induces the expression of OPG (28). Furthermore, Wnt3a exhibits an inhibitory effect on osteoclast formation, which is mediated through the canonical pathway (29). The present results also revealed that $L R R C 17$ may promote osteogenesis by increasing the Wnt3a levels in patients with INFH.

Wnt5a stimulates non-canonical Wnt signals in osteoclast precursors, and promotes RANKL-induced osteoclast precursor differentiation and JNK phosphorylation in osteoclasts. Furthermore, JNK appears to be involved in the crosstalk between RANK and Ror2-mediated signals $(30,31)$. These results suggest that Wnt5a is produced by osteoblasts, and that this promotes osteoclast differentiation through the non-canonical Wnt pathway in osteoclast precursors. Together with the present findings, LRRC17 overexpression may inhibit RANKL-induced osteoclast differentiation by inhibiting Wnt5a, while LRRC17 interference may enhance RANKL-induced osteoclast differentiation by promoting Wnt5a.

The potential mechanism on how LRRC17 regulates Wnt signaling was investigated. Proteins that contain LRR domains control a variety of physiological processes, including bone metabolism. For example, glycan and decorin are highly expressed in the extracellular bone matrix, affecting the differentiation and proliferation of osteocytes. Mice with leucine proteoglycan deficiency develop osteoporosis, which is characterized by failure to reach the peak bone mass due to decreased bone formation (8).

One of the limitations of the present study was the small number of patient samples. In future studies, the present findings require further validation in hBMSCs derived from a larger number of patients with INFH or FNF and specimens with different causes of avascular necrosis.

Another limitations of the present study is the lack of experiments in the FNF-hBMSCs; the hBMSCs from patients with FNF should be included in the LRRC17 overexpression or knockout experiments as a control. Based on the present findings that LRRC17 was decreased in INFH-hBMSCs, it can be anticipated that a similar trend of changes could be observed in the FNF-hBMSCs, with the manipulation of LRRC17 as in the INFH-hBMSCs.

In conclusion, the present study is the first to expound the pathogenesis of INFH from the perspective of bone metabolism. The modulation of the LRRC17 gene may delay or even change the process of INFH, providing a new target for the treatment of osteonecrosis of the femoral head.

\section{Acknowledgements}

Not applicable.

Funding

No funding was received.

\section{Availability of data and materials}

The datasets used and/or analyzed during the present study are available from the corresponding author on reasonable request.

\section{Authors' contributions}

DWW and DS designed and performed most of the investigation, data analysis, confirm the authenticity of all the raw data and wrote the manuscript; ZSW and QX provided pathological assistance; KW, MTX, CZH and CZ contributed to the interpretation of the data and analyses. All of the authors have read and approved the final manuscript. 


\section{Ethics approval and consent to participate}

The present study was approved by the Ethics Committee of Liaocheng People's Hospital (approval no. 2018010). All procedures performed in studies involving human participants were in accordance with the ethical standards of the institutional and/or national research committee and with the 1964 Helsinki declaration and its later amendments or comparable ethical standards. All patients who participated in this study provided a signed written informed consent.

\section{Patient consent for publication}

Not applicable.

\section{Competing interests}

All authors declare that they have no competing interests.

\section{References}

1. Mankin HJ: Nontraumatic necrosis of bone (osteonecrosis). N Engl J Med 326: 1473-1479, 1992.

2. Maillefert JF, Tavernier C, Toubeau M and Brunotte F: Non-traumatic avascular necrosis of the femoral head. J Bone Joint Surg Am 78: 473-474, 1995.

3. Yamaguchi R, Yamamoto T, Motomura G, Ikemura S and Iwamoto Y: Incidence of nontraumatic osteonecrosis of the femoral head in the Japanese population. Arthritis Rheum 63: 3169-3173, 2011.

4. Wang W, Sun QM, Zhang FQ, Zhang QL, Wang LG and Wang WJ: Core decompression combined with autologous bone marrow stem cells versus core decompression alone for patients with osteonecrosis of the femoral head: A meta-analysis. Int J Surg 69: 23-31, 2019.

5. Andriolo L, Merli G, Tobar C, Altamura SA, Kon E and Filardo G: Regenerative therapies increase survivorship of avascular necrosis of the femoral head: A systematic review and meta-analysis. Int Orthop 42: 1689-1704, 2018.

6. Mutijima E, Maertelaer VD, Deprez M, Malaise M and Hauzeur JP: The apoptosis of osteoblasts and osteocytes in femoral head osteonecrosis: Its specificity and its distribution. Clin Rheumatol 33: 1791-1795, 2014.

7. Boregowda SV, Krishnappa V, Strivelli J, Haga CL, Booker CN and Phinney DG: Basal p53 expression is indispensable for mesenchymal stem cell integrity. Cell Death Differ 25: 679-692, 2018.

8. Kim T, Kim K, Lee SH, So HS, Lee J, Kim N and Choi Y: Identification of LRRc17 as a negative regulator of receptor activator of NF-kappaB ligand (RANKL)-induced osteoclast differentiation. J Biol Chem 284: 15308-15316, 2009.

9. Wang A, Ren M, Song Y, Wang X, Wang Q, Yang Q, Liu H, Du Z, Zhang G and Wang J: MicroRNA expression profiling of bone marrow mesenchymal stem cells in steroid-induced osteonecrosis of the femoral head associated with osteogenesis. Med Sci Monit 24: 1813-1825, 2018

10. Hong N, Kim BJ, Kim CH, Baek KH, Min YK, Kim DY, Lee SH, Koh JM, Kang MI and Rhee Y: Low plasma level of leucine-rich repeat-containing 17 (LRRc17) is an independent and additive risk factor for osteoporotic fractures in postmenopausal women. J Bone Miner Res 31: 2106-2114, 2016.

11. Kim BJ, Lee SH and Koh JM: Potential biomarkers to improve the prediction of osteoporotic fractures. Endocrinol Metab (Seoul) 35: 55-63, 2020.

12. Ficat RP: Idiopathic bone necrosis of the femoral head. Early diagnosis and treatment. J Bone Joint Surg Br 67: 3-9, 1985.

13. Schmittgen TD and Livak KJ: Analyzing real-time PCR data by the comparative C(T) method. Nat Protoc 3: 1101-1108, 2008.
14. Tan SL, Ahmad TS, Selvaratnam L and Kamarul T: Isolation, characterization and the multi-lineage differentiation potential of rabbit bone marrow-derived mesenchymal stem cells. J Anat 222: 437-450, 2013.

15. Ko JY, Wang FS, Wang CJ, Wong T, Chou WY and Tseng SL: Increased dickkopf-1 expression accelerates bone cell apoptosis in femoral head osteonecrosis. Bone 46: 584-591, 2010.

16. Phetfong J, Sanvoranart T, Nartprayut K, Nimsanor N, Seenprachawong K, Prachayasittikul V and Supokawej A: Osteoporosis: The current status of mesenchymal stem cell-based therapy. Cell Mol Biol Lett 21: 12, 2016.

17. Albers J, Keller J, Baranowsky A, Beil FT, Catala-Lehnen P, Schulze J, Amling M and Schinke T: Canonical Wnt signaling inhibits osteoclastogenesis independent of osteoprotegerin. J Cell Biol 200: 537-549, 2013.

18. Tornero-Esteban P, Peralta-Sastre A, Herranz E, Rodriguez-Rodriguez L, Mucientes A, Abasolo L, Marco F, Fernandez-Gutierrez B and Lamas JR: Altered expression of wnt signaling pathway components in osteogenesis of mesenchymal stem cells in osteoarthritis patients. PLoS One 10: e0137170, 2015.

19. Duchartre Y, Kim YM and Kahn M: The Wnt signaling pathway in cancer. Crit Rev Oncol Hematol 99: 141-149, 2016.

20. Chen J, Tu X, Esen E, Joeng KS, Lin C, Arbeit JM, Rüegg MA, Hall MN, Ma L and Long F: WNT7B promotes bone formation in part through mTORC1. PLoS Genet 10: e1004145, 2014.

21. Rodda SJ and McMahon AP: Distinct roles for Hedgehog and canonical Wnt signaling in specification, differentiation and maintenance of osteoblast progenitors. Development 133: 3231-3244, 2006.

22. Krause U, Harris S, Green A, Ylostalo J, Zeitouni S, Lee N and Gregory CA: Pharmaceutical modulation of canonical Wnt signaling in multipotent stromal cells for improved osteoinductive therapy. Proc Natl Acad Sci USA 107: 4147-4152, 2010.

23. Nagayama M, Iwamoto M, Hargett A, Kamiya N, Tamamura $Y$, Young B, Morrison T, Takeuchi H,Pacifici M,Enomoto-Iwamoto M and Koyama E: Wnt/beta-catenin signaling regulates cranial base development and growth. J Dent Res 87: 244-249, 2008.

24. Tamamura Y, Otani T, Kanatani N, Koyama E, Kitagaki J, Komori T, Yamada Y, Costantini F, Wakisaka S, Pacifici M, et al: Developmental regulation of Wnt/beta-catenin signals is required for growth plate assembly, cartilage integrity, and endochondral ossification. J Biol Chem 280: 19185-19195, 2005.

25. Cho HH, Kim YJ, Kim SJ, Kim JH, Bae YC, Ba B and Jung JS: Endogenous Wnt signaling promotes proliferation and suppresses osteogenic differentiation in human adipose derived stromal cells. Tissue Eng 12: 111-121, 2006.

26. Ring L, Neth P, Weber C, Steffens $S$ and Faussner A: $\beta$-catenin-dependent pathway activation by both promiscuous 'canonical' WNT3a-, and specific 'noncanonical' WNT4- and WNT5a-FZD receptor combinations with strong differences in LRP5 and LRP6 dependency. Cell Signal 26: 260-267, 2014.

27. Zhang S, Chen X, Hu Y, Wu J, Cao Q, Chen S and Gao Y: All-trans retinoic acid modulates Wnt3A-induced osteogenic differentiation of mesenchymal stem cells via activating the PI3K/AKT/GSK3 $\beta$ signalling pathway. Mol Cell Endocrinol 422: 243-253, 2016.

28. Yamane T, Kunisada T, Tsukamoto H, Yamazaki H, Niwa H, Takada S and Hayashi SI: Wnt signaling regulates hemopoiesis through stromal cells. J Immunol 167: 765-772, 2001.

29. Takahashi N, Maeda K, Ishihara A, Uehara S and Kobayashi Y: Regulatory mechanism of osteoclastogenesis by RANKL and Wnt signals. Front Biosci (Landmark Ed) 16: 21-30, 2011.

30. Oishi I, Suzuki H, Onishi N, Takada R, Kani S, Ohkawara B, Koshida I, Suzuki K, Yamada G, Schwabe GC, et al: The receptor tyrosine kinase Ror2 is involved in non-canonical Wnt5a/JNK signalling pathway. Genes Cells 8: 645-654, 2003.

31. Sato A, Yamamoto H, Sakane H, Koyama H and Kikuchi A: Wnt5a regulates distinct signalling pathways by binding to Frizzled2. EMBO J 29: 41-54, 2010.

This work is licensed under a Creative Commons Attribution-NonCommercial-NoDerivatives 4.0 International (CC BY-NC-ND 4.0) License. 